\title{
Provision of effective information
}

\author{
An evaluation of the quality of commercially produced patient information leaflets \\ M. A. Lewis and J. T. Newton BrDent J 2006; 201: 114-117
}

\begin{abstract}
Aim A descriptive study was undertaken to assess the quality of a range of patient information leaflets produced by the British Dental Association.
\end{abstract}

Method Twenty-nine leaflets were assessed with regard to presentation, readability and quality. The topic areas covered included: treatment, self-care and disease related information. Presentation was evaluated with regard to layout, font size, typeface, use of illustrations, paper type and print colour. Readability was assessed using the Flesch reading grade and the $S M O G$ reading grade. Quality was assessed using the DISCERN tool.

Findings All leaflets scored quite well for readability, with the average SMOG Reading Grade Level being 9.10 (SD 0.80) and the average Flesch reading Grade Level being 6.18 (SD 0.83). There were, however, some areas of presentation that could be improved, specifically font size, illustration use and paper finish, which did not comply with the RNIB guidelines. Quality ratings using the DISCERN tool were low. In particular most leaflets scored poorly in setting out clear aims in the opening paragraph, in identifying sources and dates of information provided, and other sources of advice and support available. Few leaflets discussed the option of no treatment or how the treatment would affect overall quality of life. The role of shared decision making was rarely mentioned. Conclusion Patient information leaflets produced commercially are of high production quality and good readability but tend not to be patient centred.

\section{IN BRIEF}

- Patient information leaflets can be used to support oral health promotion, treatment choice and decision making in dental settings.

- Judged against standardised criteria the commercially produced patient information leaflets investigated in this study were readable and well produced, but failed to involve the patient in decision making, tended not to present the option of having no treatment and failed to reveal information sources.

- General dental practitioners and the dental team should consider the limitations of commercially produced leaflets and ensure that their communications with patients rectify the shortcomings of the leaflets.

\section{COMMENT}

It is estimated that patients remember less than a quarter of what they hear during a clinical consultation. ${ }^{1}$ Yet, in an era of patient-centred care, based on the principle of a fully informed patient actively involved in the decision-making process, provision of effective information is a prerequisite. One longstanding strategy in health education to improve knowledge has been the use of written patient information leaflets. What counts as effective? At the absolute minimum, patients must be able to read and understand the information. Do dental education and information leaflets pass this test?

Lewis and Newton set out to find out. They examined 29 commonly available patient education and information leaflets produced by the BDA. The leaflets covered aspects of dental treatment, oral self-care, and disease-related information. Each was evaluated for presentation, readability, and quality using tools validated for the assessment of patient information leaflets. The good news? Readability level was reasonable for all leaflets, and their design and presentation met many best practice criteria. However, three points were highlighted: material may need to be sourced at an easier level or in a different language where English is not the first language; some design features need to be adapted for leaflets to be useful for the elderly or sight-impaired; and finally, illustrations need to be made appropriate and sensitive to the target audience.

The bad news? All 29 leaflets were graded as low in quality meaning they had extensive shortcomings in terms of reliability and quality of information. Limitations included no reference to evidence or alternative sources of information, no information on treatment risks or the effect of choosing not to have treatment. Each of which are key elements in facilitating a successful partnership approach to care, in addition to equipping patients to give informed consent. Given the lack of prior evidence, Lewis and Newton have provided a valuable study that highlights clear concerns about the content and design of existing dental patient information leaflets. The authors put forward a number of useful recommendations for general dental practitioners as to how existing leaflets could be adapted, and most importantly draw attention to widely available guidelines for the development of new health education materials. ${ }^{2}$

Lewis and Newton's study points to the way ahead; emphasising the importance of devoting more attention to developing effective patient-orientated materials. For this to be successful, however, materials should be informed by lay expertise. One approach would be to consult patients themselves on the relevance, usefulness, and comprehensibility of written information leaflets. This is even more important when we consider the great variation in individual patient's needs and preferences as to the depth of information desired.

S. Baker

Lecturer in Psychology, School of Clinical Dentistry, Sheffield

1. Boundouki $G$, Humphris $G$, Field A. Knowledge of oral cancer, distress and screening intentions: longer term effects of a patient information leaflet. Patient Education and Counselling 2004; 53: 71-77.

2. Department of Health, Patient Information Toolkit at www.doh.gov.uk/nhsidentity

DOI: $10.1038 /$ sj.bdj.4813825 